\title{
Risk factors for venous thromboembolism in endometrial cancer
}

S. Pin $\mathrm{MD}^{* \dagger}{ }^{\dagger}$ J. Mateshaytis MD, ${ }^{*}$ S. Ghosh PhD PStat PStat (ASA), ${ }^{\dagger}$ E. Batuyong MBT, ${ }^{\ddagger}$ and J.C Easaw MD PhD ${ }^{+}$

\section{ABSTRACT}

Background Venous thromboembolism (VTE) in malignancy is associated with poor outcomes. We conducted a retrospective review of VTE in patients with endometrial cancer to characterize the VTE incidence, identify factors that contribute to VTE risk, and compare survival outcomes in patients with and without VTE.

Methods A retrospective chart review identified 422 eligible patients who underwent surgery for endometrial cancer (1 January 2014 to 31 July 2016). The primary outcome was VTE. Binary logistic regression identified risk factors for VTE; significant risk factors were included in a multivariate analysis. Kaplan-Meier estimates are reported, and log rank tests were used to compare the Kaplan-Meier curves. Risk-adjusted estimates for overall survival based on VTE were determined using a multivariate Cox proportional hazards model.

Results The incidence of VTE was $6.16 \%$ overall and $0.7 \%$ within 60 days postoperatively. Non-endometrioid histology, stages 3 and 4 disease, laparotomy, and age $(p<0.1)$ were identified as factors associated with vTE and were included in a multivariate analysis. The overall death rate in patients with VTE was $42 \%$ (9\% without VTE): hazard ratio, 5.63; 95\% confidence interval, 2.86 to 11.08; $p<0.0001$. Adjusting for age, stage of disease, and histology, risk of death remained significant for patients with a VTE: hazard ratio, $2.20 ; 95 \%$ confidence interval, 1.09 to $4.42 ; p=0.0271$.

Conclusions A method to identify patients with endometrial cancer who are at high risk for vTE is important, given the implications of VTE for patient outcomes and the frequency of endometrial cancer diagnoses. Factors identified in our study might assist in the recognition of such patients.

Key Words Venous thromboembolism, VTE, pulmonary embolism, PE, DVT, endometrial cancer, risk factors, postoperative, surgery

Curr Oncol. 2020 August:27(4)198-203

www.current-oncology.com

\section{INTRODUCTION}

Venous thromboembolism (VTE) is a term that encompasses both deep vein thrombosis and pulmonary embolism. A well-recognized complication of malignancy, VTE is associated with poor outcomes ${ }^{1}$ and is a common complication of major pelvic or abdominal surgery ${ }^{2}$. Patients with gynecologic malignancies requiring definitive surgical management are therefore at increased risk of thrombotic events. However, the incidence and risk factors associated with the development of VTE in the postoperative gynecologic oncology setting are not clearly defined, and the use of thromboprophylaxis is inconsistent.

Endometrial carcinoma is the 4th most common malignancy diagnosed in women (after breast, lung, and colorectal cancer), and many cases are curable ${ }^{3}$. The optimization of survival in the context of endometrial cancer, especially as it pertains to disease complications, is therefore paramount. According to the literature, the incidence of postoperative VTE in patients with endometrial cancer varies between $0.8 \%$ and $8.1 \%^{3-5}$. Consistently, it is observed that VTE is associated with reduced overall survival in this patient population ${ }^{6-8}$.

Guidelines suggest that patients with malignancies should receive postoperative thromboprophylaxis to reduce the risk of a thrombotic event and, subsequently, poor outcomes $^{9-11}$. In particular, an evidence-based clinical practice guideline from the American College of Chest Physicians recommends the use of extended antithrombotic therapy (28 days postoperatively) in patients undergoing open abdominal or pelvic surgery for malignancy, because those patients appear to be at the highest risk for VTE events ${ }^{11}$. Despite those strong recommendations, 
significant variability in prescribing practices remains among gynecologic oncologists. As evidence of that variability, the use of extended VTE prophylaxis (a duration of $\geq 28$ days postoperatively) was not considered the standard of care until recently in our gynecologic oncology tertiary care centre. Considering endometrial cancer specifically, Canadian guidelines for interventions such as extended VTE prophylaxis do not currently exist. With that situation in mind, the objective of our study was to investigate the incidence of, and risk factors for, VTE in patients with endometrial cancer in a setting in which extended postoperative VTE prophylaxis was not routinely administered. The ultimate goal of the study was to identify risk factors for VTE specific to that patient population, both in the immediate postoperative period and after longer-term follow up.

\section{METHODS}

Approval to proceed with the retrospective chart review was provided after assessment using the ARECCI (A Project Ethics Community Consensus Initiative) tool. Eligible patients were identified using an ACATs (Adult Coding Access Targets for Surgery) code for uterine cancer (WU08), and those who underwent surgery for endometrial cancer at the Royal Alexandra Hospital in Edmonton, Alberta, between 1 January 2014 and 31 July 2016 were included. All patients with gynecologic cancer in northern Alberta (population of approximately 2 million) receive their surgery at that centre. Patients were excluded from the analysis if they had benign pathology, a non-endometrial primary malignancy, recurrent disease, or a known vTE before surgery.

Dual therapy with a sequential compression device and short-course thromboprophylaxis was routinely used in the postoperative period. However, extended thromboprophylaxis beyond discharge from hospital was given at the discretion of the surgeon. Patients were followed from the date of surgery to the first symptomatic VTE event, death, or 24 months from the index date, whichever came first. Data were collected through multiple electronic medical records including Netcare (the provincial electronic medical record system), Cancer Surgery Alberta, and the provincial cancer database, ARIA. A diagnosis of VTE was made based on a symptomatic clinical presentation. Each patient was assessed for 129 different endpoints, including demographics, pathology, surgical details, vTE (timing, type), anticoagulation, lab work, chemotherapy, and survival. The primary outcome of the study was the incidence of VTE, assessed in both the immediate postoperative period and after longer-term follow-up.

Descriptive statistics are reported for the study variables: means with standard deviation for normally distributed continuous variables, medians with range for non-normally distributed continuous variables, and frequencies and proportions for categorical variables. Chisquare tests were used to compare categorical variables, and the Fisher exact test is reported for cell frequencies less than 5. Binary logistic regression was then used to identify the variables that might represent possible risk factors for VTE. The variables were tested by univariate logistic regression, and variables significant at $p<0.10$ were considered for a multivariate model.
The multivariate logistic regression used combinations of variables that had proved to be significant in the univariate analysis and that had previously been identified in the literature as correlated with vTE ${ }^{12-18}$. We elected to maintain both laparotomy and histology as variables in the multivariate analysis despite demonstrated nonsignificance in the univariate analysis, because both are considered to be clinically relevant. Overall survival was calculated from date of the procedure to date of death (the date of last follow-up was used for patients who were alive at the end of the study period). Kaplan-Meier estimates and corresponding 95\% confidence intervals are reported. Log-rank tests were used to compare the Kaplan-Meier curves. A Cox proportional hazards model was used to determine the risk factors associated with overall survival. Hazard ratios and corresponding 95\% confidence intervals are reported. All statistical analyses were conducted using the SAS software application (version 9.3: SAS Institute, Cary, NC, U.S.A.). A $p$ value less than 0.05 was used for statistical significance.

\section{RESULTS}

\section{Baseline Population Characteristics}

Of the 422 analyzed patients, 26 experienced VTE events postoperatively, resulting in an incidence rate of $6.2 \%$. The incidence of VTE in the postoperative period (within 60 days of surgery) was $0.7 \%$. Table I describes baseline population characteristics. The two groups (VTE and no-VTE) showed no significant differences with respect to age and body mass index. The length of hospital stay was similar in the groups, with $95 \%$ of the patients having a hospital stay of less than 5 days' duration. The median length of stay was 2 days for the VTE group and 1 day for the no-VTE group.

Based on a review of the literature, several putative risk factors for VTE were evaluated in this patient population. Elevated serum cancer antigen $125(>35 \mathrm{U} / \mathrm{mL})$ was noted in $15.4 \%$ of the VTE group and $8.6 \%$ of the no-VTE group. Thrombocytosis was similar in the groups: $7.7 \%$ in the VTE group and $8.1 \%$ in the no-VTE group. Compared with patients who did not experience a VTE, those who did experience a VTE were more likely to have either stage 3 or 4 disease (based on the most recent 2009 Fédération Internationale de Gynécologie et d'Obstétrique staging for endometrial cancer): $57.7 \%$ compared with $21 \%{ }^{19}$. Higher-risk histologies (serous, clear cell, and undifferentiated, among others) were seen in $57.7 \%$ of patients experiencing VTE and in $27 \%$ of those not experiencing VTE. The predominant type of surgery also differed between the groups: $53 \%$ of the VTE group, but only $32 \%$ of the non-VTE group, received open surgery (laparotomy). In most cases, estimated blood loss was less than $500 \mathrm{~mL}$, regardless of which group the patients belonged to. Finally, Eastern Cooperative Oncology Group performance statuses of $0-3$ were comparably distributed between the groups (Table I).

While in hospital, patients in our study received dual-therapy prophylaxis with short-course postoperative thromboprophylaxis and a sequential compression device. The use of extended thromboprophylaxis $(\geq 28$ days postoperatively) was not the standard of care during the study period and was therefore given at the discretion of the surgeon. Based on our assessment of the available 
TABLE I Baseline characteristics of the study population

\begin{tabular}{|c|c|c|}
\hline \multirow[t]{2}{*}{ Variable } & \multicolumn{2}{|c|}{ Venous thromboembolism } \\
\hline & Yes & No \\
\hline Participants [n (\%)] & $26(6.2)$ & $396(93.8)$ \\
\hline \multicolumn{3}{|l|}{ Age (years) } \\
\hline Median & 69 & 62 \\
\hline Range & $53-84$ & $27-90$ \\
\hline \multicolumn{3}{|l|}{ Body mass index } \\
\hline$<30[n(\%)]$ & $11(42.3)$ & $154(38.9)$ \\
\hline$\geq 30[n(\%)]$ & $15(57.7)$ & $242(61.1)$ \\
\hline Median value & 30.8 & 32.9 \\
\hline \multicolumn{3}{|l|}{ Length of stay } \\
\hline$\leq 5$ Days $[n(\%)]$ & $22(95.7)$ & $364(95.3)$ \\
\hline$>5$ Days $[n(\%)]$ & $1(4.3)$ & $18(4.7)$ \\
\hline Median (days) & 2 & 1 \\
\hline \multicolumn{3}{|c|}{ Cancer antigen 125 [n (\%)] } \\
\hline$<35 \mathrm{U} / \mathrm{mL}$ & $22(84.6)$ & $362(91.4)$ \\
\hline$\geq 35 \mathrm{U} / \mathrm{mL}$ & $4(15.4)$ & $34(8.6)$ \\
\hline \multicolumn{3}{|l|}{ Platelets $[n(\%)]$} \\
\hline$<400 \times 10^{9} / \mathrm{L}$ & $24(92.3)$ & 364 (91.9) \\
\hline$\geq 400 \times 10^{9} / \mathrm{L}$ & $2(7.7)$ & $32(8.1)$ \\
\hline \multicolumn{3}{|l|}{ Stage $[n(\%)]$} \\
\hline I & $9(34.6)$ & $278(70.2)$ \\
\hline II & $2(7.7)$ & $35(8.8)$ \\
\hline III & $10(38.5)$ & $72(18.2)$ \\
\hline IV & $5(19.2)$ & $11(2.8)$ \\
\hline \multicolumn{3}{|c|}{ Endometrioid grade $[n(\%)]$} \\
\hline $1-2$ & $8(72.7)$ & $258(89.3)$ \\
\hline 3 & $3(27.3)$ & $31(10.7)$ \\
\hline \multicolumn{3}{|l|}{ Histology $[n(\%)]$} \\
\hline Endometrioid & $11(42.3)$ & $289(73)$ \\
\hline Serous & $8(30.8)$ & $53(13.4)$ \\
\hline Sarcoma & $6(23.1)$ & $29(7.3)$ \\
\hline Clear cell & $1(3.8)$ & $14(3.5)$ \\
\hline Mixed & $0(0)$ & $1(0.3)$ \\
\hline Undifferentiated & $0(0)$ & $7(1.8)$ \\
\hline Other ${ }^{\mathrm{a}}$ & $0(0)$ & $3(0.7)$ \\
\hline \multicolumn{3}{|l|}{ Type of surgery [n (\%)] } \\
\hline Laparotomy & $14(53.8)$ & $127(32.1)$ \\
\hline Laparoscopy & $12(46.2)$ & $269(67.9)$ \\
\hline \multicolumn{3}{|c|}{ Estimated blood loss $[n(\%)]$} \\
\hline$\leq 500 \mathrm{~mL}$ & $21(100)$ & $336(98)$ \\
\hline$>500 \mathrm{~mL}$ & $0(0)$ & $7(2)$ \\
\hline \multicolumn{3}{|l|}{ ECOG PS $[n(\%)]$} \\
\hline 0 & $17(68)$ & $269(71.4)$ \\
\hline 1 & $6(24)$ & $89(23.6)$ \\
\hline 2 & $2(8)$ & $16(4.2)$ \\
\hline 3 & $0(0)$ & $3(0.8)$ \\
\hline
\end{tabular}

a Other: Adenocarcinoma not identified as endometrioid adenocarcinoma. ECOG PS = Eastern Cooperative Oncology Group performance status.

data, 368 patients received a short course of anticoagulation postoperatively while in hospital (87.2\%). Extended VTE prophylaxis (for at least 28 days postoperatively) was prescribed for 28 patients $(6.6 \%)$. When those patients were further assessed, more than half $(57.1 \%)$ were found to have been on lifelong anticoagulation for another reason before surgery, and they were subsequently continued on their anticoagulation dose postoperatively. Of those who developed a VTE, only $7.7 \%$ ( 2 of 26 ) had received extended VTE prophylaxis ( $\geq 28$ days postoperatively).

\section{Clinical Variables Associated with Postoperative VTE and Predictive Model}

A univariate analysis assessed variables that might be potential risk factors for VTE in the postoperative setting (Table II). The variables found to be significant included stage 3 or 4 disease [odds ratio (OR): 2.3 ; $95 \%$ confidence interval (CI): 1.51 to $3.41 ; p<0.0001$ ], non-endometrioid histology (OR: 1.9 ; 95\% CI: 1.28 to $2.88 ; p=0.0016$ ), laparotomy (OR: $1.6 ; 95 \%$ CI: 1.05 to $2.34 ; p=0.0265$ ), and age as a continuous variable (OR: $1.1 ; 95 \%$ CI: 1.02 to $1.1 ; p=0.0046$ ). Those variables were then further assessed in a multivariate analysis, with the aim of identifying independent risk factors for VTE in this patient population. We used a logistic regression analysis with a binary outcome (VTE Vs. no-VTE). Table III summarizes the results. In the multivariate analysis, stages 3 and 4 disease (OR: $1.9 ; 95 \%$ CI: 1.24 to $3.03 ; p=0.0035$ ) and age at procedure (OR: $1.06 ; 95 \%$ CI: 1.01 to $1.10 ; p=0.0118$ ) remained statistically significant; non-endometrioid histology (OR: $1.3 ; 95 \%$ CI: 0.85 to $2.08 ; p=0.2150$ ) and laparotomy (OR: $1.4 ; 95 \%$ CI: 0.87 to 2.09 ; $p=0.1771$ ) did not.

\section{Survival}

We observed increased mortality in patients who developed blood clots after surgery. The risk of death (all-cause mortality) in the VTE group was 5 times that in the no-VTE group (hazard ratio: 5.63 ; 95\% CI: 2.86 to $11.08 ; p<0.0001$ ). At 12 months postoperatively, the survival probability was $84 \%$ for those with a VTE compared with $95 \%$ for those without; at 24 months postoperatively, those probabilities were reduced to $58 \%$ and $92 \%$ (Figure 1 ).

With respect to the timing of thrombotic events after surgery in patients with endometrial cancer, $12 \%$ occurred by postoperative day 60 , and $50 \%$ occurred by postoperative day 180. In the multivariate Cox proportional hazards model used to determine the risk-adjusted estimates of VTE for overall survival (adjusted for histology, stage, and age at diagnosis), the hazard for death in the VTE group was 2.2 times that in non-VTE group (hazard ratio: 2.20 ; $95 \%$ CI: 1.09 to $4.42 ; p=0.0271$ ).

\section{DISCUSSION}

We identified several variables that correlate with an increased risk of a VTE developing in patients with endometrial cancer who have received surgical treatment. Specifically, increased age, stages 3 and 4 disease, non-endometrioid histology, and laparotomy are all associated with an increased risk of postoperative VTE. Increasing age and more advanced stage of malignancy are both commonly associated with an increase in VTE in many cancers ${ }^{12-15}$. As in our study, others have reported that a high-risk histology type contributes to VTE risk $^{16}$. Histology proved statistically significant in the univariate analysis, but lost significance 
TABLE II Univariate logistic regression analysis of variables potentially associated with postoperative venous thromboembolism in patients with endometrial cancer

\begin{tabular}{|c|c|c|c|c|}
\hline Variable & Comparator & OR & $95 \% \mathrm{Cl}$ & $p$ Value \\
\hline \multicolumn{5}{|l|}{ Cancer antigen 125} \\
\hline$\leq 35 \mathrm{U} / \mathrm{mL}$ & $>35 \mathrm{U} / \mathrm{mL}$ & 1.4 & 0.79 to 2.44 & 0.2485 \\
\hline \multicolumn{5}{|l|}{ Stage of disease } \\
\hline I-II & III-IV & 2.3 & 1.51 to 3.41 & $<0.0001$ \\
\hline \multicolumn{5}{|l|}{ Platelet count } \\
\hline$\leq 400$ & $>400$ & 0.97 & 0.46 to 2.05 & 0.9438 \\
\hline \multicolumn{5}{|l|}{ Grade } \\
\hline $1-2$ & 3 & 1.7 & 0.86 to 3.41 & 0.1240 \\
\hline \multicolumn{5}{|l|}{ Histology } \\
\hline Endometrioid & Non-endometrioid & 1.9 & 1.28 to 2.88 & 0.0016 \\
\hline \multicolumn{5}{|l|}{ Length of stay } \\
\hline$\leq 5$ Days & $>5$ Days & 0.96 & 0.34 to 2.68 & 0.9361 \\
\hline \multicolumn{5}{|l|}{ Type of surgery } \\
\hline Laparoscopy & Laparotomy & 1.6 & 1.05 to 2.34 & 0.0265 \\
\hline \multicolumn{5}{|l|}{ ECOG PS } \\
\hline 0 & $1-4$ & 1.17 & 0.49 to 2.80 & 0.7203 \\
\hline \multicolumn{5}{|l|}{$\mathrm{BMI}$} \\
\hline$\leq 30$ & $>30$ & 0.93 & 0.62 to 1.39 & 0.7295 \\
\hline Age at procedure & & 1.1 & 1.02 to 1.1 & 0.0046 \\
\hline
\end{tabular}

a Continuous variable.

$\mathrm{OR}=$ odds ratio; $\mathrm{Cl}=$ confidence interval; ECOG PS = Eastern Cooperative Oncology Group performance status; $\mathrm{BMI}=$ body mass index .

TABLE III Multivariate regression model for postoperative venous thromboembolism in patients with endometrial cancer

\begin{tabular}{lcccc}
\hline \multicolumn{1}{c}{ Variable } & Comparator & OR & 95\% CI & p Value \\
\hline $\begin{array}{l}\text { Histology } \\
\text { Endometrioid }\end{array}$ & Non-endometrioid & 1.3 & 0.85 to 2.08 & 0.2150 \\
$\begin{array}{l}\text { Type of surgery } \\
\text { Laparoscopy }\end{array}$ & Laparotomy & 1.4 & 0.87 to 2.09 & 0.1771 \\
$\begin{array}{l}\text { Stage of disease } \\
\text { I-II }\end{array}$ & III-IV & 1.9 & 1.24 to 3.03 & 0.0035 \\
Age at procedure & & 1.06 & 1.01 to 1.10 & 0.0118 \\
\hline
\end{tabular}

a Continuous variable.

$\mathrm{OR}=$ odds ratio; $\mathrm{Cl}=$ confidence interval.

when analyzed as part of the multivariate regression model. That result is likely secondary to the number of events. Given the importance of differentiating the high-risk histology types because of known differences in survival, maintaining that variable as part of the multivariate assessment was felt to be clinically relevant.

The link between laparotomy and the development of VTE is well described in the literature ${ }^{17,18}$. Although our study data demonstrated the significance of laparotomy in the univariate analysis (as it pertains to VTE risk), that significance was lost in the multivariate analysis. We suspect that the loss of significance is likely related to the small number of VTE events in the present study, particularly in the immediate postoperative period. Additionally, the fact that most patients (67\%) underwent laparoscopic surgery could have affected the significance of laparotomy as it pertains to VTE risk. However, given laparotomy's clinical significance and the evidence of its importance in the literature, maintaining it in the multivariate analysis was deemed appropriate. By combining variables that were statistically significant in the univariate analysis in a multivariate approach, we identified a number of factors that might assist in identifying patients with endometrial cancer who are at high risk for development of a VTE.

We found that thromboembolic events in patients with endometrial cancer are associated with increased mortality. That observation is consistent with previous studies of malignancy and the effect of VTE on survival ${ }^{7,19-21}$. We must recognize that our data analysis assessed all-cause mortality and not mortality specific to VTE itself. By virtue of the components of the multivariate analysis, it might be expected that the "high-risk patients" (advanced malignancy, older age, more aggressive histology, requirement for adjuvant therapy) would have higher mortality in general, 


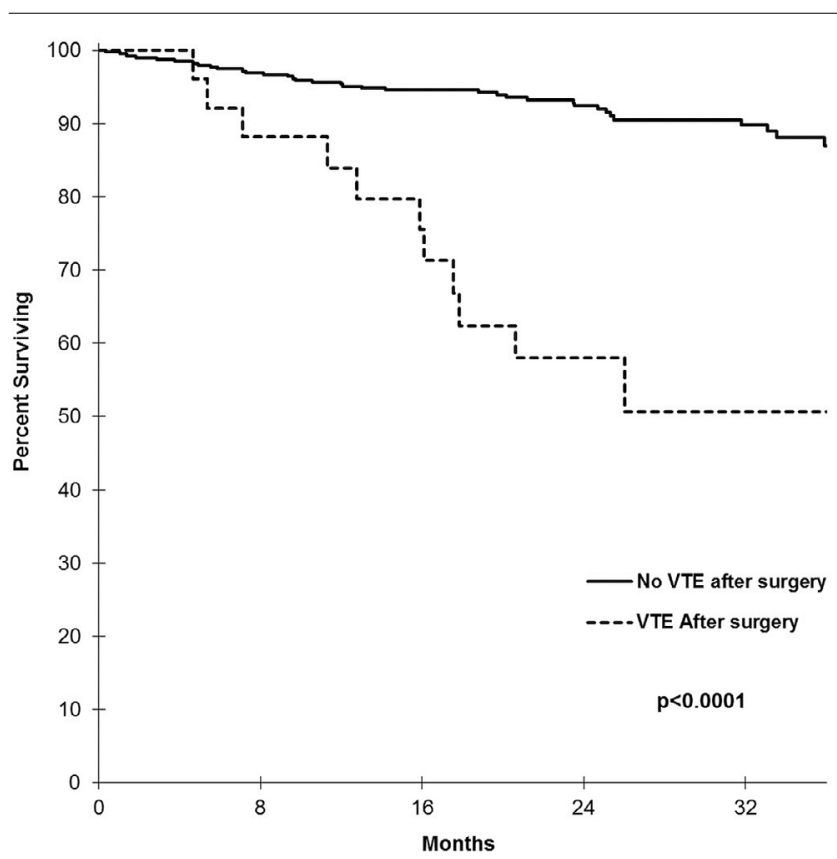

FIGURE 1 Postoperative survival probabilities (all-cause mortality) for patients with endometrial cancer who did and did not develop venous thromboembolism (VTE). At 12 months postoperatively, the survival probability for patients developing VTE was $84 \%$; it was $95 \%$ for patients not developing a VTE. At 24 months postoperatively, the rates were $58 \%$ and $92 \%$. The overall postoperative death rate (all-cause mortality) for patients with endometrial cancer who experienced a thrombotic complication was $42 \%$ (11 of 26 ); the death rate was $9 \%$ for patients who did not develop a VTE (36 of 396). The risk for death in the VTE group was thus almost 5 times that in the no-VTE group: hazard ratio, 5.63; $95 \%$ confidence interval, 2.86 to $11.08 ; p<0.0001$. In our analysis, $12 \%$ of VTE events ( 3 of 26 ) occurred by postoperative day 30 , and $50 \%$ (13 of 26) occurred by postoperative day 180 .

regardless of VTE status. When risk was adjusted for stage of disease, histology type, and age, VTE was shown to be an independent predictor of mortality (the hazard for death in the VTE group was 2.2 times that in the non-VTE group). Interestingly, in evaluating the timing of the VTE events, only $12 \%$ occurred in the immediate postoperative period (within 60 days of surgery); $50 \%$ occurred within 180 days of surgery. That observation suggests that only a portion of the VTE events are likely to be directly related to surgical status; perhaps the others are more significantly associated with other patient or disease factors. Determining the direct effect of VTE on survival in this patient population is therefore difficult. Late VTE events also raise the question of the effect of adjuvant therapy after surgical intervention on the development of VTE in patients with endometrial cancer. That question warrants further investigation, particularly given that the general link between chemotherapy and development of VTE is supported by the literature ${ }^{22,23}$. We believe that the variables we identified as correlating with the incidence of VTE could serve as a useful tool to assist physicians in identifying the patients with endometrial cancer most at risk for VTE.

A significant strength of our study is the number of variables reviewed in the assessment of potential risk factors for VTE. All told, we evaluated 129 different endpoints for each patient. Another strength is the fact that the variables ultimately identified as being associated with an increased risk of developing a VTE (stage of disease, histology, type of surgery, and age) can all be objectively measured, making them useful in the clinical context.

One of the important limitations of our study is the relatively small number of VTE events seen; ultimately, we observed VTEs in only $6.16 \%$ of patients (26 VTE events in 422 patients). When evaluating the VTE rate in the immediate postoperative period, our clot rate was found to be $0.7 \%$. We believe that the small number of VTE events ultimately affected our results. First, we anticipate that, with a larger number of VTE events, we might have identified more events occurring in the immediate postoperative period. We also believe that the type of surgery likely affected the number of VTE events (specifically, a greater proportion of our patients underwent laparoscopic rather than open surgery). We did find that laparotomy was significantly associated with VTE in the univariate analysis; however, that significance was lost in the multivariate analysis. We suspect that, with an increased number of VTE events, we would find that statistical significance emerges in the multivariate analysis as well, which would accord with the links between laparotomy and VTE established in the literature.

The retrospective nature of our study is an additional, important limitation. The pre-existing data used in a retrospective study is inherently associated with a potential for bias. For example, we had intended to review serum albumin as a variable that might contribute to VTE risk in our patient population, because serum albumin has been demonstrated to be a clinically important variable in terms of disease progression, prognosis, and severity ${ }^{24}$. Unfortunately, those data points were not collected for most patients.

One area of interest for further research and evaluation would be the use of extended thromboprophylaxis in laparotomy compared with laparoscopic surgery for endometrial cancer. At present, the evidence for the routine use of extended prophylaxis in the laparoscopic setting is lacking ${ }^{25,26}$. However, the 2019 guideline from the American Society of Clinical Oncology suggests that the use of extended prophylaxis (up to 4 weeks) should be considered in patients undergoing either major abdominal or pelvic surgery for malignancy with high-risk features or additional risk factors for VTE (regardless of the mode of surgery-laparoscopy or laparotomy $)^{27}$. However, that recommendation is not specific to endometrial cancer. Evidently, further research in this area is necessary.

Another question raised by the present study is the concept of VTE in the immediate postoperative period. We demonstrated a $0.7 \%$ incidence of VTE within 60 days of surgical intervention, compared with an overall rate of $6.16 \%$. It would be interesting to determine, in a study with a larger number of VTE events, whether that postoperative VTE rate is consistent, and if so, whether changes to postoperative VTE prophylaxis alter the risk in a measurable way.

It is possible that the factors identified in our study could ultimately be used in a predictive model to identify patients with endometrial cancer who are at high risk for VTE. To develop such a model, the variables identified here would have to be tested in a larger population with a higher number of VTE events to validate the data. Subsequently, a prospective study 
would be necessary if the goal is to create a predictive model for postoperative VTE in patients with endometrial cancer.

\section{CONCLUSIONS}

Postoperative VTE prophylaxis for patients with endometrial cancer is inconsistently prescribed in various treatment centres. Some practice guidelines recommend extended prophylaxis; however, that recommendation is not specific to patients with endometrial cancer. Patients treated at our centre were rarely given extended VTE prophylaxis $(>91 \%$ did not receive extended prophylaxis). The frequency with which endometrial cancer is diagnosed, the relative "curability" of the disease, and the effect of VTE on morbidity and mortality together raise the question of whether there is a rationale to prescribe extended prophylaxis to all patients in the postoperative setting-and potentially continuing that prophylaxis long-term in patients at high risk. However, given the relative infrequency of a VTE diagnosis in this patient population $(6.2 \%)$, it is also reasonable to ask whether all patients with endometrial cancer should receive postoperative extended thromboprophylaxis, because that therapy also carries potential risk (particularly bleeding).

Our study identified 4 risk factors for vTE in the relevant patient population: age, stage of disease, histology, and type of surgery. Those risk factors might be useful in identifying "patients at high risk" who might ultimately benefit from ongoing VTE prophylaxis. The data from the present study are hypothesis-generating and will require prospective validation in a larger population. The study has raised a number of important questions that each warrant further research.

\section{CONFLICT OF INTEREST DISCLOSURES}

We have read and understood Current Oncology's policy on disclosing conflicts of interest, and we declare that we have none.

\section{AUTHOR AFFILIATIONS}

*Obstetrics and Gynecology, University of Alberta, Edmonton, ${ }^{\dagger}$ Cross Cancer Institute, Edmonton, and ${ }^{\ddagger}$ Tom Baker Cancer Centre, University of Calgary, Calgary, AB.

\section{REFERENCES}

1. Sorensen HT, Mellemkjaer L, Olsen JH, Baron JA. Prognosis of cancers associated with venous thromboembolism. NEngl J Med 2000;343:1846-50.

2. Turpie AGG, Chin BSP, Lip GYH. Venous thromboembolism: pathophysiology, clinical features, and prevention. $B M J$ 2002;325:887-90.

3. Matsuo K, Yessaian AA, Lin YG, et al. Predictive model of venous thromboembolism in endometrial cancer. Gynecol Oncol 2013;128:544-51.

4. Rauh-Hain JA, Hariton E, Clemmer J, etal. Incidence and effects on mortality of venous thromboembolism in elderly women with endometrial cancer. Obstet Gynecol 2015;125:1362-70.

5. Graul A, Latif N, Zhang X, et al. Incidence of venous thromboembolism by type of gynecologic malignancy and surgical modality in the National Surgical Quality Improvement Program. Int J Gynecol Cancer 2017;27:581-7.

6. Khorana AA, Francis CW, Culakova E, Kuderer NM, Lyman GH. Thromboembolism is a leading cause of death in cancer patients receiving outpatient chemotherapy. J Thromb Haemost 2007;5:632-4.

7. Khorana AA. Cancer and coagulation. Am J Hematol 2012; 87(suppl 1):S82-7.

8. Rodriguez AO, Gonik AM, Zhou H, Leiserowitz GS, White RH.
Venous thromboembolism in uterine cancer. Int J Gynecol Cancer 2011;21:870-6.

9. American College of Obstetricians and Gynecologists (ACOG). Prevention of deep vein thrombosis and pulmonary embolism. ACOG practice bulletin no. 84. Obstet Gynecol 2007;110:429-40.

10. Clarke-Pearson DL, Barber EL. Venous thromboembolism in gynecologic surgery: are we any closer to determining an optimal prophylaxis regimen? Gynecol Oncol 2015;138:495-6.

11. Gould MK, Garcia DA, Wren SM, et al. Prevention of VTE in nonorthopedic surgical patients: antithrombotic therapy and prevention of thrombosis, 9th ed: American College of Chest Physicians evidence-based clinical practice guidelines. Chest 2012;141(suppl):e227S.

12. Silverstein MD, Heit JA, Mohr DN, Petterson TM, O'Fallon WM, Melton LJ3rd. Trends in the incidence of deep vein thrombosis and pulmonary embolism: a 25-year population-based study. Arch Intern Med 1998;158:585-93.

13. Naess IA, Christiansen SC, Romundstad P, Cannegieter SC, Rosendaal FR, Hammerstrøm J. Incidence and mortality of venous thrombosis: a population-based study. J Thromb Haemost 2007;5:692-9.

14. Chew HK, Wun T, Harvey D, Zhou H, White RH. Incidence of venous thromboembolism and its effect on survival among patients with common cancers. Arch Intern Med 2006;166:458-64.

15. Sallah S, Wan JY, Nguyen NP. Venous thrombosis in patients with solid tumors: determination of frequency and characteristics. Thromb Haemost 2002;87:575-9.

16. Blom JW, Osanto S, Rosendaal FR. The risk of a venous thrombotic event in lung cancer patients: higher risk for adenocarcinoma than squamous cell carcinoma. J Thromb Haemost 2004;2:1760-5.

17. Jorgensen EM, Li A, Modest AM, Leung K, Moore Simas TA, Hur HC. Incidence of venous thromboembolism after different modes of gynecologic surgery. Obstet Gynecol 2018;132:1275-84.

18. Qu H, Li Z, Zhai Z, et al. Predicting of venous thromboembolism for patients undergoing gynecological surgery. Medicine (Baltimore) 2015;94:1653.

19. Bhatla N, Aoki D, Sharma DN, Sankaranarayanan R. Cancer of the cervix uteri. Int J Gynecol Obstet 2018;143(suppl 2):22-36.

20. Lyman GH, Culakova E, Poniewierski MS, Kuderer NM. Morbidity, mortality and costs associated with venous thromboembolism in hospitalized patients with cancer. Thromb Res 2018;164(suppl 1):S112-18.

21. Donnellan E, Khorana AA. Cancer and venous thromboembolic disease: a review. Oncologist 2017;22:199-207.

22. Heit JA, Silverstein MD, Mohr DN, Petterson TM, O'Fallon WM, Melton LJ. Risk factors for deep vein thrombosis and pulmonary embolism: a population-based case-control study. Arch Intern Med 2000;160:809-15.

23. Zahir MN, Shaikh Q, Shabbir-Moosajee M, Jabbar AA. Incidence of venous thromboembolism in cancer patients treated with cisplatin based chemotherapy—a cohort study. BMC Cancer 2017;17:57.

24. Nazha B, Moussaly E, Zaarour M, Weerasinghe C, Azab B. Hypoalbuminemia in colorectal cancer prognosis: nutritional marker or inflammatory surrogate? World J Gastrointest Surg 2015;7:370-7.

25. Freeman AH, Barrie A, Lyon L, et al. Venous thromboembolism following minimally invasive surgery among women with endometrial cancer. Gynecol Oncol 2016;142:267-72.

26. Bouchard-Fortier G, Geerts WH, Covens A, Vicus D, Kupets $\mathrm{R}$, Gien LT. Is venous thromboprophylaxis necessary in patients undergoing minimally invasive surgery for gynecologic malignancy? Gynecol Oncol 2014;134:228-32.

27. Key NS, Khorana AA, Kuderer NM, et al. Venous thromboembolism prophylaxis and treatment in patients with cancer: ASCO clinical practice guideline update.JClin Oncol 2020;38:496-520. 